\title{
A skin-over-liquid platform with compliant microbumps actuated by pyro-EHD pressure
}

\author{
O. Gennari ${ }^{1}$, R. Rega', M. Mugnano ${ }^{1}$, E. Oleandro ${ }^{1,2}$, L. Mecozzi ${ }^{1}$, V. Pagliarulo ${ }^{1}$, E. Mazzon ${ }^{3}$, A. Bramanti ${ }^{1,3}$, A. Vettoliere ${ }^{1}$, \\ C. Granata', P. Ferraro' ${ }^{1}$ and S. Grilli'
}

\begin{abstract}
The unique deformability and the compliance ability of thin sheets on soft substrates attract much interest for studying the phenomena related to elastic instabilities as well as for sensing very weak forces such as those generated by live cells in vitro. However, the techniques used currently for producing such platforms are affected by a high degree of complexity and poor repeatability. Moreover, their deformability is usually used as a passive response to the action of an external force. Herein we propose a novel concept for a reliable and dynamic skin-over-liquid system made of a periodic array of highly compliant microbumps actuated through electrode-free electrohydrodynamic (EHD) pressure. We demonstrate that these structures are highly repeatable and capable of swelling and deflating easily under a simple thermal stimulation driven by the pyroelectric effect, thus providing a challenging platform that can be actively controlled at the microscale. Furthermore, we show the proof of principle by swelling these microbumps for mechanically stimulating live cells in vitro, thus opening the route to more reliable and easy to accomplish assays in the field of mechanobiology.
\end{abstract}

\section{Introduction}

The deformability of thin sheets on soft substrates has attracted much interest in the last decades due to the possibility of studying, with high accuracy, all of those physical phenomena related to wrinkling, buckling, or other mechanical instabilities ${ }^{1,2}$. Currently, polydimethylsiloxane (PDMS) can be considered the material that best matches the softness requirements for these types of studies and is widely used in the literature. PDMS is a silicone-based component introduced by Whitesides and co-workers in the '90s for the rapid fabrication of microfluidic devices, through the so-called "soft lithography"3,4. The key advantages of PDMS include low

Correspondence: S Grilli (s.grilli@isasi.cnr.it)

${ }^{1}$ Institute of Applied Sciences and Intelligent Systems of the National Research Council (CNR-ISASI), Via Campi Flegrei 34, 80078 Pozzuoli (NA), Italy

${ }^{2}$ Department of Mathematics and Physics, University of Campania "L. Vanvitelli", Viale Lincoln 5, 81100 Caserta, Italy

Full list of author information is available at the end of the article. costs, short fabrication and turn-around time, optical transparency, flexibility and bio-compatibility, thus making it a first choice in various fields such as microfluidics, sensors, micro-optics, and microbiology ${ }^{5-10}$.

The wrinkling effect occurring on the surface of PDMS has been presented in the literature for a wide variety of applications. Rogers and coworkers ${ }^{11}$ used pre-strained PDMS for producing stretchable forms of silicon, while other groups have exploited the wrinkling effect for producing stretchable metal electrodes ${ }^{12,13}$. Both the mechanical behavior and the wavelength distribution of the buckling patterns have been investigated deeply to promote the applications of such structures ${ }^{14-18}$. Duan et al. ${ }^{19}$ have studied in particular the buckling modes of micro/nanowires on elastomeric PDMS to identify the strategies for producing buckled structures in a selected manner. The wrinkling effect has also been used for producing optical components such as microlens arrays and mechanically tunable diffusers ${ }^{20,21}$. Nagashima et al. ${ }^{22}$ 
used the gratings produced by skin folding for driving the alignment of DNA filaments, while hierarchical wrinkles have also been produced for particle manipulation applications $^{23}$. The common feature in all of these techniques is the use of pre-strained cross-linked PDMS in combination with oxygen plasma treatment to achieve a thin silica-like skin on the surface of a soft elastomeric substrate. Very recently, the PDMS substrate has been replaced by polystyrene-based materials able to shrink upon annealing treatments to produce wrinkled surfaces with super hydrophobic and adjustable adhesion properties $^{24,25}$.

Another family of buckling structures also attracts much interest due to the high degree of compliance and consists of skin-over-liquid systems ${ }^{26-29}$. The wrinkling effects occurring on the skin under compression are investigated deeply for studying the physical phenomena related to elastic instabilities ${ }^{27,28}$. Moreover, the high deformability and compliance of the skin make it sensitive to very weak forces, such as those generated by live cells in vitro. In fact, Harris's group presented seminal demonstrations of the use of a skin-over-liquid system for observing cellular traction forces ${ }^{29}$. They used a smart heat treatment of the PDMS layer for producing a crosslinked skin floating onto the liquid silicone underneath. Successively, Huang et al. ${ }^{27}$ presented an alternative technique where the skin was a PS film floating over a liquid layer of water. All of these skin-over-liquid structures were revolutionary and provided the first evidence of cellular traction forces, but unfortunately, they are produced by complicated procedures affected by poor repeatability, thus limiting the development and their applications.

Here, we present a novel dynamic platform made of highly compliant microbumps (CMB) actuated by a simple thermal stimulation. For the first time, we develop a skin-over-liquid system with a surface that can be switched easily and reversibly between a flat state and a topographic state consisting of an array of periodic microbumps. Moreover, these bumps exhibit a high degree of deformability due to the liquid film under the skin, thus opening the route to a radically new field of highly sensitive mechanobiology. The wrinkle-based studies mentioned above make use of solid-over-solid systems that, depending on the specific procedure, produce wrinkle patterns with different characteristics. Here, instead, we do not propose a new method for wrinkle patterns, but we use for the first time a solid-over-liquid platform able to swell reversibly into a periodic array of microbumps with a smooth surface.

We apply a standard oxygen plasma treatment to a layer of silicone to form a very thin silica-like skin on the surface $^{30}$, with the final advantage of exposing a solid surface (skin) that is highly compliant due to the liquid underneath (silicone). Moreover, we actuate reversibly the shape of the skin with high repeatability into an array of microbumps by a heat-controlled pyro-EHD pressure. The fabrication technique is rapid and easy to accomplish since it makes use of a periodically poled lithium niobate (PPLN) crystal that drives a liquid dielectrophoresis (LDEP) effect ${ }^{31,32}$, by using simply the strong electric field generated on its surface through the pyroelectric effect. To the best of our knowledge, the liquid silicone has never been used under direct oxidation conditions. In fact, all of the studies mentioned above deal with the deformability of thin solid sheets onto solid substrates, such as crosslinked PDMS or polystyrene-based layers. Here, instead, we show that the oxidized fresh silicone gives rise to a skin-over-liquid platform, which forms an amazing structure resembling that of a water mattress at the microscale level when actuated by pyro-EHD pressure, thus leading us to call it a "silicone mattress." The results show how such CMBs can change their shape very easily with a high deformation range, thus suggesting their great potential for inducing mechanical stimulation at the microscale for reliable and easy to accomplish mechanobiology studies.

\section{Materials and methods \\ Silicone}

The PPLN crystals were spin coated with a thin layer of commercial silicone, namely, the base component of Sylgard 184 (Dow Corning), usually used for fabricating elastomeric structures.

\section{PPLN crystals}

The LN crystals were bought from Crystal Technology Inc. in the form of both sides polished $500-\mu \mathrm{m}$-thick $c$-cut 3 -inch wafers and cut into square samples $(2 \times 2) \mathrm{cm}^{2}$ in size by a standard diamond saw. After solvent cleaning and resist patterning, the samples were subjected to electric field poling. Two types of PPLN structures were used here: (1) a square array of hexagonal domains at $200-\mu \mathrm{m}$ period; and (2) linear domains at approximately $400-\mu \mathrm{m}$ period.

\section{Silicone oxidation}

After spin-coating with fresh silicone, the PPLN crystals were exposed directly to an $\mathrm{O}_{2}$ plasma treatment (Atto low pressure plasma system, Diener Electronic, Germany). Plasma exposure was performed in a cylindrical discharge chamber made of borosilicate glass with a length of $30 \mathrm{~cm}$ and an inner diameter of $211 \mathrm{~mm}$. The system was pumped with a two-stage oil rotary pump with a pumping speed of $2.5 \mathrm{~m}^{3} / \mathrm{h}$. The base pressure was a few Pascals. Plasma was created with an inductively coupled RF generator, operating at a frequency of $40 \mathrm{kHz}$ and a nominal power of approximately $200 \mathrm{~W}$. Commercially available 
oxygen was leaked into the discharge chamber. Samples were introduced in the chamber and processed after a vacuum time of $5 \mathrm{~min}$. One cycle of plasma treatment was 15 min long.

\section{The heating system}

The heating system was obtained by integrating a titanium coil on the lower side of the PPLN crystal, so a circulating current produces strictly local heating due to the Joule effect. The resistive coil, in a circular shape, was fabricated by using standard optical photolithography and thin film deposition via dc sputtering in an ultra-high vacuum system (basic pressure of $1.2 \cdot 10^{-5} \mathrm{~Pa}$ ). A subsequent lift-off process defined the final geometry, resulting in a $T i$ circular coil having a thickness of $250 \mathrm{~nm}$. The loop radius and the coil width are 9 and $1 \mathrm{~mm}$, respectively, and the resistance is $80 \Omega$ at room temperature. Therefore, a circulating current of $10 \mathrm{~mA}$ produces a dissipative power of $8 \mathrm{~mW}$.

\section{Fibroblast cell culture}

The mouse embryonic fibroblast NIH/3T3 cells were purchased from ECACC (Sigma-Aldrich, Milan, Italy). They were routinely grown in Dulbecco's modified Eagle's medium (DMEM) containing $4.5 \mathrm{~g} / \mathrm{L}$ D-glucose and supplemented with 10\% FBS (fetal bovine serum) (GIBCO, Gaithersburg, MD, USA), 100 units $/ \mathrm{mL}$ penicillin, and $100 \mu \mathrm{g} / \mathrm{mL}$ streptomycin. Subsequently, they were harvested from the tissue culture flasks by incubation with a $0.05 \%$ trypsin/EDTA solution for $5 \mathrm{~min}$. The cells were then centrifuged, resuspended in a complete medium, and then seeded on the samples at a density of $1 \times$ $10^{5}$ cells $/ \mathrm{mL}$. The NIH/3T3 cells were then incubated in conventional 50-mm-diameter Petri dishes at $37^{\circ} \mathrm{C}$ under a humidified $5 \% \mathrm{CO}_{2}$ atmosphere. The cells were harvested and seeded onto the CMB platform by using the CYTOOchamber ${ }^{\mathrm{TM}}$ and observed under a standard inverted optical microscope (AxioVert, Carl Zeiss, Jena, Germany). The CYTOOchamber allowed us to mount the PPLN crystal equipped with the heating system and perform the pyroelectric stimulation during culturing.

\section{Immunofluorescence}

The cells were cultured for $24 \mathrm{~h}$ on the surface of interest and then fixed by standard procedures. The cells were then stained by Alexa fluor 488 phalloidin and blue fluorescent Hoechst 33342 dye, trihydrochloride trihydrate (Molecular Probes Invitrogen) for visualizing nuclei and actin filaments.

\section{Results and discussion}

Figure 1 shows the schematic view of the process steps (see Materials and methods for more details).
A PPLN crystal with hexagonal reversed ferroelectric domains $^{33}$ is first spin coated with fresh silicone (i.e., without curing agents) (Fig. 1a) and then oxidized by a 15min-long reaction with $\mathrm{O}_{2}$ plasma (Fig. $1 \mathrm{~b}$ ). The reactive oxygen radicals attack the surface of the silicone layer and replace the methyl groups $\left(-\mathrm{CH}_{3}\right)$ bonded to $\mathrm{Si}$ atoms with $\mathrm{O}$ atoms, generating $\mathrm{SiO}_{\mathrm{x}}$ bonds corresponding to a stiff and very thin skin, which cannot be assimilated to a true silica material and is commonly referred to as the "silica-like" layer ${ }^{30,34,35}$. The insets in Fig. 1a, b show the side view of a water droplet on the surface of the silicone layer before and after the plasma treatment, respectively, with the indication of the measured contact angle. These results clearly show the highly hydrophilic nature of the skin, in full agreement with other studies reported in the literature $^{34}$. Therefore, once exiting the plasma chamber, the liquid silicone exposes a very thin solid skin on the top (Fig. 1c), with high hydrophilicity and high deformability due to the liquid phase underneath. The effect is similar to that leading to the formation of the cream-skin on the surface of boiling milk when blowing cold air upon it. The sample is then observed under a conventional optical microscope while heated up to approximately $35^{\circ} \mathrm{C}$ to stimulate the pyroelectric effect (see Fig. 1d).

It is well known that under equilibrium conditions at room temperature, the charge of the spontaneous polarization $P_{\mathrm{s}}$ of a $c$-cut wafer of LN crystal is balanced by the screening charges on the surface, thus producing an electrically neutral system ${ }^{31-36}$, as shown schematically in Fig. 1c. The thermal treatment makes the $P_{\mathrm{s}}$ intensity change according to $\Delta P_{i}=p_{i} \Delta T$, where $P_{i}$ is the coefficient of the polarization vector, $p_{i}$ is the pyroelectric coefficient, and $\Delta T$ is the temperature variation. In particular, the polarization intensity decreases under heating, so a transient condition arises where the screening charges are no more compensated by the polarization charge of the crystal (see the scheme in Fig. 1d). In particular, the regions inside the hexagons exhibit an excess of positive charges, while the regions surrounding the hexagons present an excess of charges with a negative polarity. Due to the high pyroelectric coefficient of $\mathrm{LN}$, the resulting electric field is non-negligible and follows the periodicity of the reversed domains, with minimum values across the hexagonal boundaries and maximum values inside and outside the hexagons ${ }^{31-33}$, as shown schematically by the simplified profile in Fig. 1d. Therefore, the silicone is subjected to an L-DEP force $\left(F_{\mathrm{L}-\mathrm{DEP}}\right)$ according to the Helmoltz equation ${ }^{37}$ for a dielectric and incompressible liquid:

$$
F_{L-D E P}=1 / 2 E^{2}\left(\varepsilon-\varepsilon_{0}\right)
$$

where $E$ is the electric field intensity, and $\varepsilon$ and $\varepsilon_{0}$ are the permittivity of silicone and air, respectively. The stronger 
(a)

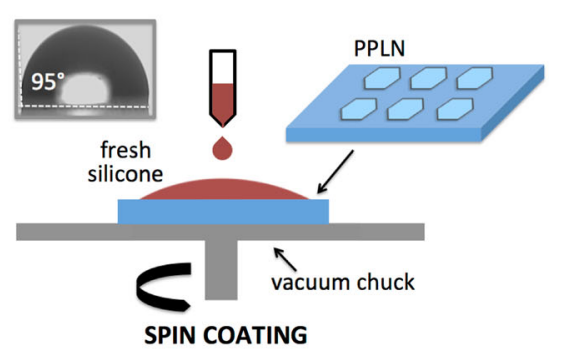

(b)

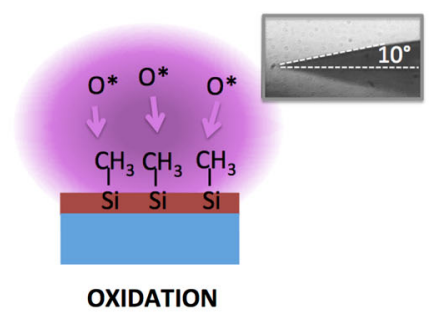

(d)

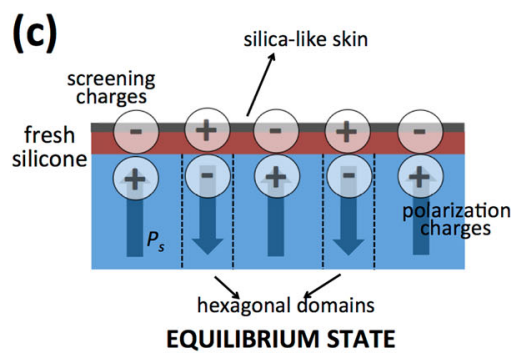

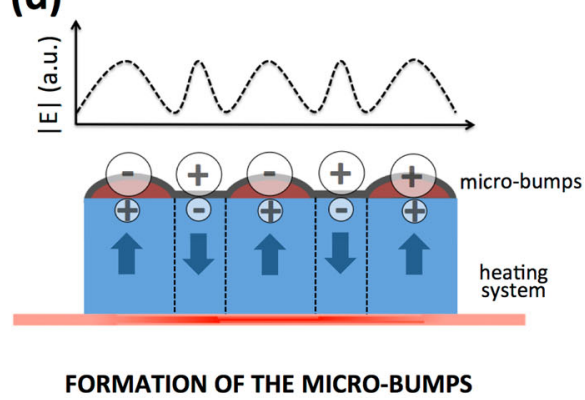

Fig. 1 Schematic illustration of the process steps a Spin-coating of a PPLN crystal with fresh silicone; $\mathbf{b}$ oxidation by a 15-min-long reaction with $\mathrm{O}_{2}$ plasma; $\mathbf{c}$ extraction from the plasma chamber of the hybrid skin-over-liquid platform and equilibrium between polarization charges in the crystal and screening charges on the surface; $\mathbf{d}$ Heating process generating uncompensated charges and a strong electric field profile on the surface of the PPLN crystal with a subsequent L-DEP effect, leading to the formation of microbumps. $P_{\mathrm{s}}$ is the spontaneous polarization of the $c$-cut LN crystal. The insets in $\mathbf{a}$ and $\mathbf{b}$ show the side view of a water droplet on the surface of the silicone layer before and after the oxidation, respectively, with the indication of the contact angle value

electric field inside and outside the hexagons and the lager permittivity of the liquid make $F_{\mathrm{L}-\mathrm{DEP}}$ move and accumulate the silicone away from the hexagonal boundaries. This mechanism produces an innovative skin-over-liquid system with "compression areas" in the hexagons and "swell areas" outside, following the periodicity of the domains with high fidelity. Since the area inside the hexagons is much smaller than that outside, the corresponding silicone accumulation is negligible, as is well shown by the following figures.

The resulting structure is a periodic array of microbumps with a high compliance capability, whose appearance is similar to that observed typically in a water mattress, but with an actuation capability. The great innovation here is due to several aspects: (1) these bumps expose a ready to use surface that is solid (i.e., the silicalike skin) but with a deformability that is much higher than that provided by the well-established platforms based on cross-linked PDMS, due to the perfectly liquid phase of the silicone layer underneath; (2) moreover, the skin here is hydrophilic and ready to use for cell culturing, in contrast to the above-mentioned PDMS structures that usually require additional steps of chemical functionalization to promote cell adhesion; (3) the high deformability opens the route to highly sensitive mechanobiology studies; (4) the bumps are repeatable and periodic, thus making the platform ideal for high-throughput studies in mechanobiology; (5) the platform is active, meaning that the bumps can be actuated reversibly by switching the skin from flat to a bumped state, and vice versa, by a simple thermal stimulation, thus enabling future dynamic studies in mechanobiology; and (6) last but not least, the fabrication procedure does not require sophisticated and expensive technologies, thus making it suitable to be implemented easily in a biology laboratory.

Supporting Movie 1 shows the typical formation of a periodic array of CMBs on a PPLN crystal during heating up to $35^{\circ} \mathrm{C}$ and observed under an optical microscope. The skin is clearly flat at room temperature in the initial part of the movie. The temperature rises progressively, and the electric field generated pyroelectrically makes the hexagonal domains appear through the transparent silicone due to an electro-optic-based mechanism ${ }^{31-33}$. The regions inside the hexagons become dark rapidly due to the compression of the skin under the action of the L-DEP effect. The skin exhibits a good mechanical strength, and the resulting traction makes it wrinkle along the square array directions, without any signs of breaking. Once the skin compression inside the hexagons is completed, the array of CMBs appears clearly in the final part of the movie. For the sake of clarity, we engraved a $\mathrm{CMB}$ platform by a conventional scalpel to show its 

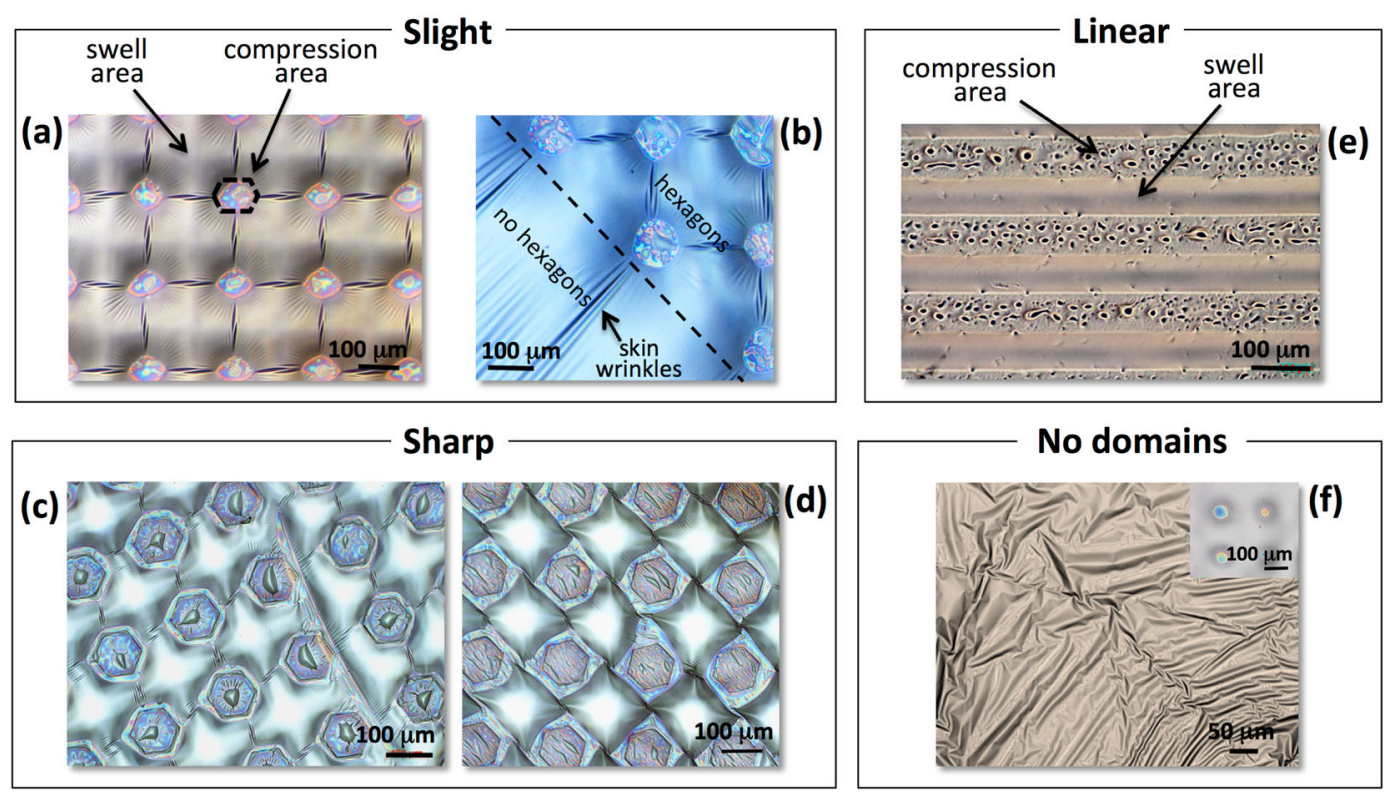

Fig. 2 Optical microscope images of typical platforms of CMBs a, $\mathbf{b}$ Slight CMBs with compression areas at 200- $\mu$ m period obtained by a silicone thickness of $20 \mu \mathrm{m}$, under two different magnifications; c, d Sharp CMBs with compression areas at approximately $200-\mu \mathrm{m}$ period obtained by a silicone thickness of $8 \mu \mathrm{m}$, observed at two different compression phases; e Linear CMBs obtained by using linear reversed domains. The image in b was taken across the boundary between two regions of the crystal with and without hexagonal domains, as indicated by the labels. The image in $\mathbf{f}$ corresponds to the result obtained in the case of a virgin crystal, namely, without reversed domains. The inset in $\mathbf{f}$ refers to a layer of mineral oil after $\mathrm{O}_{2}$ plasma treatment and L-DEP on PPLN

solid-over-liquid nature whereby, once broken, the skin makes the liquid silicone underneath leak (see Supporting Movie 2). After reaching a new equilibrium condition of the charges at the crystal/silicone interface, the electric field vanishes, and as a consequence, the compression effect into the hexagons weakens and the skin recovers the initial flat state. The intriguing feature is that the process is fully reversible and can be activated indefinitely through the thermal cycles induced by the heating plate. The compliance ability of this platform is clearly visible in Supporting Movie 3 recorded under an optical microscope while blowing slightly upon it. At each puff shot, the skin of the microbumps wrinkles visibly under the action of the weak air pressure and then recovers the original shape rapidly.

The thickness of the silicone layer is crucial and allows us to produce platforms of CMBs with three main different shapes that we call here "slight", "sharp", and "linear", as shown by the typical optical microscope images in Fig. 2a-e. The silicone thickness was approximately $20 \mu \mathrm{m}$ (spin-coating at $4000 \mathrm{RPM}$ ) in the case of the slight shape and approximately $8 \mu \mathrm{m}$ (spin-coating at 10,000 RPM) in the case of the sharp shape. Both the slight and sharp shapes consist of arrayed CMBs at approximately $200-\mu \mathrm{m}$ period, but with compression areas that are regularly inscribed into the hexagons or circumscribing them, respectively (see Fig. 2a, d).
Conversely, the thickness of the silica-like skin is not crucial and formed spontaneously on the top of the silicone during the plasma treatment.

Considering the same electric field distribution, in the case of the thicker layer $(20 \mu \mathrm{m})$, the liquid resistance is higher and the resulting compression areas appear inscribed into the hexagons (see the labels in Fig. 2a). The opposite occurs in the case of the thinner layer $(8 \mu \mathrm{m})$, where the compression areas circumscribe the hexagons, as is clearly visible in Fig. 2c, d. This means that in the case of narrow compression areas, the skin is pinched slightly by the hexagons, and the swell areas exhibit a relatively low curvature, producing continuous bumps with the liquid silicone in communication between adjacent bumps. Conversely, the large compression areas enhance the curvature of the skin in the swell areas, up to producing well-separated bumps. The picture in Fig. 2c was recorded at an intermediate temporal phase during the formation of the final shape in Fig. $2 d$ to show the evolution of the skin compression into the hexagons. The picture in Fig. 2b was recorded across a boundary between two areas of the crystal with and without hexagons (see the labels) to show the compliance nature of the platform with the skin that wrinkles under the action of the compression areas and appears like a stretched sheet. The linear shape is obtained simply by using a PPLN crystal with linear reversed domains. Moreover, in this case, the 
swell and compression areas follow with high fidelity the domain boundaries underneath (Fig. 2e).

As a control experiment, Fig. $2 \mathrm{f}$ shows the spontaneous wrinkling of the skin in the case of a single domain LN crystal, namely, without the ferroelectric domain pattern. The skin wrinkles according to a random redistribution of the liquid underneath when exiting the plasma chamber. These wrinkles are out of the scope of this work and demonstrate here simply that the LN ferroelectric pattern is of crucial importance for the formation of the bumps. Another control example is shown in the inset in Fig. 2f, where we used a layer of mineral oil over the PPLN crystal, instead of silicone. The L-DEP effect is clearly visible, but without the skin formation, due to the lack of $\mathrm{Si}$ atoms in the liquid layer. In fact, the skin-over-liquid structure is possible only by using a Si-based material that is liquid at room temperature.

We used an interferometer in digital holography (DH) modality for measuring the dynamic evolution of the three-dimensional (3D) shape of the CMBs during formation. Due to the contact-free nature of this technique, we were able to reconstruct the shaping of the bumps during heating, without any mechanical perturbation. Details about this optical technique can be found in refs. ${ }^{38,39}$. Figure 3a shows the schematic view of the optical set-up, based on a Mach-Zehnder interferometer.

The CW laser beam at a wavelength of $532 \mathrm{~nm}$ is spilt into two beams and properly expanded. The object beam is collected by a microscope objective after passing the sample so that a focused image is obtained on the image plane. The reference beam impinges directly on the CCD camera sensor (hologram plane), where the out of focus image of the sample is superimposed by a beam-splitter. On the sensor plane, an interference pattern (the hologram) is generated by the optical interference between the object beam and the reference beam. The hologram holds information about the refractive index and the thickness of the sample, which modulate both the amplitude and the phase of the transmitted object beam. The hologram was recorded and processed by numerical techniques, which allowed us to recover the complex optical field on the image plane of the microscope objective. Figure 3b, c show the typical surface images of the CMBs reconstructed by DH at 23 and $50^{\circ} \mathrm{C}$, respectively, while the chart in Fig. 3d shows the corresponding profiles retrieved at three different temperatures along a direction passing through the center of the bumps. Supporting Movie 4 shows the temporal evolution of the $\mathrm{CMB}$ surface reconstructed by $\mathrm{DH}$ while heating from room temperature to $50^{\circ} \mathrm{C}$. The results clearly show the homogeneous and regular shaping of the bumps up to an approximately $40-\mu \mathrm{m}$ height (Fig. 3d). It is important to note that the shaping phenomenon lasts for approximately $1 \mathrm{~s}$ and is completed just at $35^{\circ} \mathrm{C}$, beyond which no additional swelling occurs. This operation temperature is of fundamental importance because it makes the CMB platform highly suitable for biological applications where typically temperatures up to approximately $36^{\circ} \mathrm{C}$ are considered safe for the cell vitality. Moreover, the electrode-free generation of the electric field distribution greatly favors the implementation of the technique under a standard optical microscope without cumbersome voltage generators and control circuits.

It is well known that the cell adhesion strength and mechanical stimulation are of fundamental importance for a wide variety of studies and applications ${ }^{40-46}$. Here, we cultured the $\mathrm{CMB}$ platforms with live fibroblast cells to demonstrate the possibility of using these actuable structures for stimulating live cells in vitro mechanically at the microscale. See the Materials and methods section for details about the cell culture. Figure $3 \mathrm{e}$, f show the optical microscope images of a single microbump under deflated and swollen states, respectively, with a live fibroblast cell adhering on the surface. These images clearly show the high deformability of the microbump that appears squeezed by the action of the cell filopodia during swelling. The platform is handled easily under standard culture conditions without losing its structural integrity. To better elucidate the material-cytoskeleton crosstalk during adhesion, we fixed the cells after a 24-h incubation and performed immunofluorescence reactions (see Materials and methods for details). We performed three replicates of the experiment, and Fig. $3 g$, h show the typical images recorded on a glass slide, used as a control, and on the $\mathrm{CMB}$ platform, respectively. The images clearly demonstrate how the cells seeded on the CMB are able to assemble their cytoskeleton in a different way when subjected to mechanical stress, compared to the cells seeded on the control slide (glass). The cells on the glass slide exhibit round nuclei (stained blue) and a wellorganized actin structure with a normal polymerization of the actin stress fibers (stained green) (see Fig. 3g). Conversely, the cells cultured on the CMB platform appear significantly polarized and elongated with an irregular formation of the actin stress fibers. They are smaller than the cells on the control and exhibit nuclei that are elongated along the polarization direction (see Fig. 3h).

It is important to note that the interaction between the cell and the bump is active, meaning that the swelling area exerts a dynamic pressure on the cell. The compliance ability would be of great advantage for mechanotransduction studies because the forces are easily transferred, either from the cells to the substrate or vice versa. Compared to traditional glass substrates widely used for mechano-transduction studies due to easy access for cell culturing, the relief topography of the CMBs provides the great advantage of studying cells in a 3D dynamic environment. Moreover, considering the ease of 

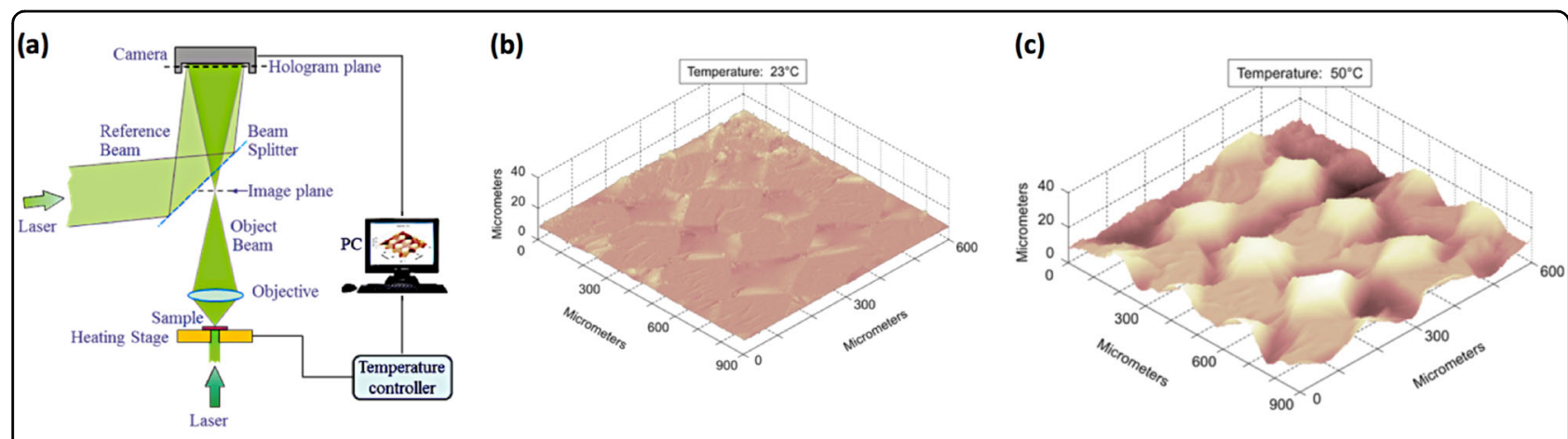

(d)
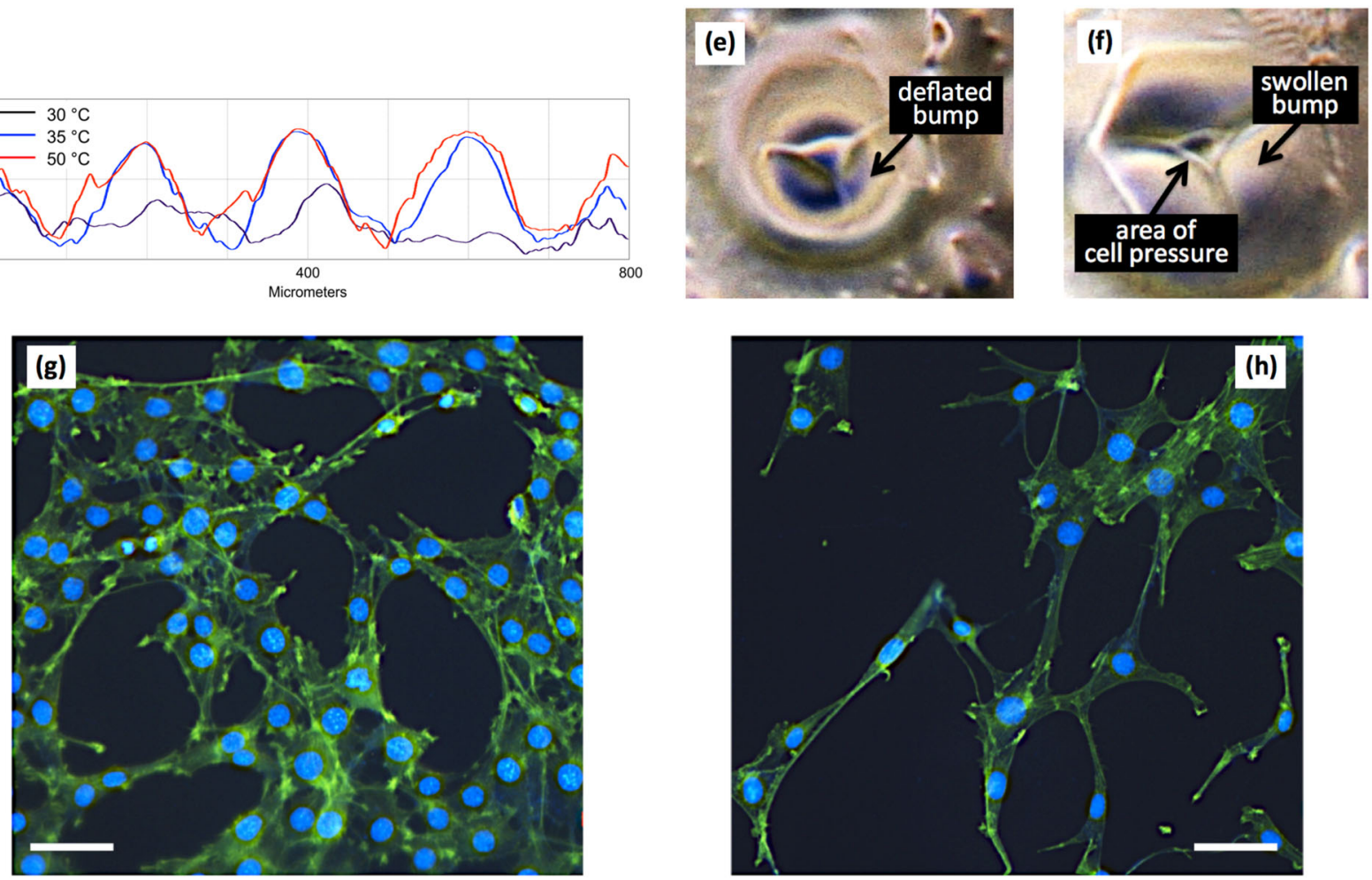

Fig. 3 Deflated and swollen states. a Schematic view of the DH set-up used for evaluating the profile of typical CMBs during shaping up to approximately $50^{\circ} \mathrm{C} ; \mathbf{b}$, c two images of the reconstructed surface shapes at 23 and $50{ }^{\circ} \mathrm{C}$, respectively; $\mathbf{d}$ typical linear profiles of the $\mathrm{CMBs}$ at three different temperatures; $\mathbf{e}, \mathbf{f}$ optical microscope images of a single microbump under deflated and swollen states, respectively, while cultured in a Petri dish with live fibroblast cells; $\mathbf{g}, \mathbf{h}$ immunofluorescence images of the cells cultured on a standard glass slide and on the bumps after a 24-h incubation, respectively. The scale bar is 50 mm

implementation, the technique would be directly applicable in a biological laboratory without expensive and complicated equipment.

\section{Conclusion}

We show here how the pyro-EHD pressure, acting on a layer of oxidized liquid silicone, is able to actuate easily and rapidly an array of compliant microbumps embedded into an innovative skin-over-liquid platform. These structures have the peculiarity of being periodic, exposing a stiff skin, and being highly compliant due to the liquid silicone flowing underneath. Furthermore, the effect is fully reversible, thus achieving a flexible and switchable platform. We believe that the high compliance and the actuation abilities are of particular interest for mechanobiology applications. In fact, the preliminary results here clearly show the ability of the CMB platform to induce a significant variation in the nuclei and stress fiber organization, thus opening the route to systematic studies on mechanical cues that control the interaction of cells with their surrounding environment over a range of compliance never studied before, with a significant impact in the field of tissue engineering through neural stem cells. 


\section{Acknowledgements}

We thank the IRCCS Centro Neurolesi "Bonino Pulejo" for financial support and trainee Chiara Del Giorno for help with the experimental activities. We also thank the Italian Ministry of Education University and Research for the financial support of an innovative research doctor for industrial characterization within the XXXIII cycle doctorate of the University Vanvitelli.

\section{Author details}

${ }^{1}$ Institute of Applied Sciences and Intelligent Systems of the National Research Council (CNR-ISASI), Via Campi Flegrei 34, 80078 Pozzuoli (NA), Italy. ${ }^{2}$ Department of Mathematics and Physics, University of Campania "L. Vanvitelli", Viale Lincoln 5, 81100 Caserta, Italy. ${ }^{3}$ IRCCS Neurolesi "Bonino Pulejo", Messina, Italy

\section{Conflict of interest}

The authors declare no conflict of interest.

\section{Publisher's note}

Springer Nature remains neutral with regard to jurisdictional claims in published maps and institutional affiliations.

Supplementary information is available for this paper at https://doi.org/ 10.1038/s41427-018-0100-z.

Received: 5 March 2018 Revised: 5 September 2018 Accepted: 27 October 2018.

Published online: 23 January 2019

\section{References}

1. Genzer, J. \& Groenewold, J. Soft matter with hard skin: From skin wrinkles to templating and material characterization. Soft Matter 2, 310 (2006).

2. Chung, J. Y., Nolte, A. J. \& Stafford, C. M. Surface wrinkling: A versatile platform for measuring thin-film properties. Adv. Mater. 23, 349-368 (2011).

3. Xia, Y. \& Whitesides, G. M. Soft lithography. Annu. Rev. Mater. Sci. 28, 153-184 (1998).

4. Berthier, E., Young, E. W. K. \& Beebe, D. Engineers are from PDMS-land, biologists are from polystyrenia. Lab. Chip. 12, 1224 (2012).

5. Sackmann, E. K., Fulton, A. L. \& Beebe, D. J. The present and future role of microfluidics in biomedical research. Nature 507, 181 (2014).

6. Weibel, D. B., Di Luzio, W. R. \& Whitesides, G. M. Microfabrication meets microbiology. Nat. Rev. Microbiol. 5, 209 (2007).

7. Unger, M. A., Chou, H. P., Thorsen, T., Scherer, A. \& Quake, S. R. Monolithic microfabricated valves and pumps by multilayer soft lithography. Science $\mathbf{2 8 8}$ 113 (2000).

8. Miccio, L., Memmolo, P., Grilli, S. \& Ferraro, P. All-optical microfluidic chips for reconfigurable dielectrophoretic trapping through SLM light induced patterning. Lab. Chip. 12, 4449 (2012).

9. Xi, X., Zhao, D., Tong, F. \& Cao, T. The self-assembly and patterning of thin polymer films on pyroelectric substrates driven by electrohydrodynamic instability. Soft Matter 8, 298 (2012).

10. Park, S. et al. Silicones for streatchable and durable soft devices: beyond Sylgard-184. ACS Appl. Mater. Intefaces 10, 11261 (2018).

11. Khang, D. -Y., Jiang, H., Huang, Y. \& Rogers, J. A. A stretchable form of singlecrystal silicon for high-performance electronics on rubber substrates. Science 311, 208 (2006)

12. Tang, J. et al. Highly stretchable electrodes on wrinkled polydimethylsiloxane substrates. Sci. Rep. 5, 16527 (2015).

13. Ahn, S. H. \& Guo, L. J. Spontaneous formation of periodic nanostructures by localized dynamic wrinkling. Nano Lett. 10, 4228 (2010).

14. Kim, P., Abkarian, M. \& Stone, H. A. Hierarchical folding of elastic membranes under biaxial compressive stress. Nat. Mater. 10, 952 (2011).

15. Jiang, $\mathrm{H}$. et al. Finite deformation mechanics in buckled thin films on compliant supports. Proc. Natl Acad. Sci. USA 104, 15607-15612 (2007).

16. Sanii, B., Smith, A. M., Butti, R., Brozell, A. M. \& Parikh, A. N. Bending membranes on demand: Fluid phospholipid bilayers on topographically deformable substrates. Nano Lett. 8, 866 (2008).

17. Park, J. Y. et al. Controlled wavelength reduction in surface wrinkling of poly (dimethylsiloxane). Soft Matter 6, 677 (2010).
18. Stafford, C. M. et al. A buckling-based metrology for measuring the elastic moduli of polymeric thin films. Nat. Mater. 3, 545 (2004).

19. Duan, Y. Q., Huang, Y. A. \& Yin, Z. P. Competing buckling of micro/nanowires on compliant substrates. J. Phys. D. Appl. Phys. 48, 045302-045309 (2015).

20. Ohzono, T., Suzuki, K., Yamaguchi, T. \& Fukuda, N. Tunable optical diffuser based on deformable wrinkles. Adv. Opt. Mater. 1, 374-380 (2013).

21. Chan, E. P. \& Crosby, A. J. Fabricating microlens arrays by surface wrinkling. Adv. Mater. 18, 3238-3242 (2006)

22. Nagashima, S. et al. Spontaneous formation of aligned DNA nanowires by capillarity-induced skin folding. Proc. Natl Am. Sci. 114, 6233 (2017).

23. Efimenko, K. et al. Nested self-similar wrinkling patterns in skins. Nat. Mater. 4 293 (2005).

24. Scarratt, L. R. J., Hoatson, B. S., Wood, E. S., Hawkett, B. S. \& Neto, C. Durable superhydrophobic surfaces via spontaneous wrinkling of teflon AF. ACS Appl. Mater. Interfaces 8, 6743 (2016).

25. Owais, A., Smith-Palmer, T., Gentle, A. \& Neto, C. Influence of long-range forces and capillarity on the function of underwater superoleophobic wrinkled surfaces. Soft Matter 14, 6627 (2018)

26. Huang, R. \& Suo, Z. Very thin solid-on-liquid structures: the interplay of flexural rigidity, membrane force, and interfacial force. Thin Solid Films. $\mathbf{4 2 9}$ 273 (2003).

27. Huang, J. et al. Capillary wrinkling of floating thin polymer films. Science 317, 650 (2007).

28. Huang, J., Davidovitch, B., Santangelo, C. D., Russell, T. P. \& Menon, N. Smooth cascade of wrinkles at the edge of a floating elastic film. Phys. Rev. Lett. 105, 038302 (2010).

29. Harris, A. K., Stopak, D. \& Wild, P. Fibroblast traction as a mechanism for collagen morphogenesis. Nature 290, 249 (1981).

30. Hillborg, $\mathrm{H}$. et al. Crosslinked polydimethylsiloxane exposed to oxygen plasma studied by neutron reflectometry and other surface specific techniques. Polym. (Guildf.). 41, 6851-6863 (2000).

31. Ferraro, P., Grilli, S., Miccio, L. \& Vespini, V. Wettability patterning of lithium niobate substrate by modulating pyroelectric effect to form microarray of sessile droplets. Appl. Phys. Lett. 92, 213107 (2008)

32. Grilli, S., Vespini, V. \& Ferraro, P. Surface-charge lithography for direct PDMS micro-patterning. Langmuir 24, 13262-13265 (2008).

33. Grilli, S., Paturzo, M., Miccio, L. \& Ferraro, P. In situ investigation of periodic poling in congruent $\mathrm{LiNbO}_{3}$ by quantitative interference microscopy. Meas. Sci. Technol. 19, 074008 (2008).

34. Befahy, S. et al. Thickness and elastic modulus of plasma treated PDMS silicalike surface layer. Langmuir 26, 3372 (2010).

35. Rhee, D., Lee, W. -K. \& Odom, T. W. Crack-free, soft wrinkles enable switchable anisotropic wetting. Angew. Chem. Int. Ed. 56, 6523 (2017).

36. Carrascosa, M., Garcia-Cabanes, A., Jubera, M., Ramiro, J. B. \& Agullo-Lopez, F. LiNbO3: A photovoltaic substrate for massive parallel manipulation and patterning of nano-objects. Appl. Phys. Rev. 2, 040605 (2015).

37. Jones, T. B. Liquid dielectrophoresis on the microscale. J. Electrost. 51, 290 (2001).

38. Ferraro, P. et al. Phase map retrieval in digital holography: avoiding the undersampling effect by a lateral shear approach. Opt. Lett. 32, 2233 (2007).

39. Di Caprio et al. Quantitative label-free animal sperm imaging by means of digital holographic microscopy. IEEE J. Sel. Top. Quant. Electron. 16, 833 (2010).

40. Hyuna, J., Chenb, J., Settonb, L. A. \& Chilkoti, A. Patterning cells in highly deformable microstructures: Effect of plastic deformation of substrate on cellular phenotype and gene expression. Biomaterials 27, 1444-1451 (2006).

41. Beningo, K. A. \& Wang, Y. L. Flexible substrata for the detection of cellular traction forces. Trends Cell Biol. 12, 79 (2002).

42. Yang, Y., Kulangara, K., Lam, R. T. S., Dharmawan, R. \& Leong, K. W. Effects of topographical and mechanical property alterations induced by oxygen plasma modification on stem cell behavior. ACS Nano 6, 8591 (2012).

43. Polacheck, W. J. \& Chen, C. S. Measuring cell-generated forces: a guide to the available tools. Nat. Methods 13, 415 (2016).

44. Park, J. S. et al. Directed migration of cancer cells guided by the graded texture of the underlying matrix. Nat. Mater. 15, 792 (2016).

45. Campàs, O. et al. Quantifying cell-generated mechanical forces within living embryonic tissues. Nat. Methods 11, 183 (2014).

46. Tan, J. L. et al. Cells lying on a bed of microneedles: An approach to isolate mechanical force. Proc. Natl Acad. Sci. USA 100, 1484 (2003). 